\title{
Lesões Epidérmicas pela Síndrome de Stevens Johnson: Cicatrização com Uso de Membrana de Celulose e Ácidos Graxos
}

\author{
Epidermal Injuries by Stevens-Johnson Syndrome: \\ Healing Following the use of Cellulose Membrane and Fatty Acids
}

\author{
Lesiones Epidérmicas por el Síndrome de Stevens Johnson: \\ Cicatrización con el uso de Membrana de Celulosa y Ácidos Grasos
}

\author{
Ana Cristina Geiss Casarolli', Halana Batistel Barbosa², \\ Lara Adrianne Garcia Paiano ${ }^{3}$, Luciana Magnani Fernandes ${ }^{3}$, Thaís Dresch Eberhardt ${ }^{1}$
}

\begin{abstract}
RESUMO
A Síndrome de Stevens Johnson pode ser definida como uma afecção inflamatória aguda, febril e autolimitada, que afeta a pele e a membrana mucosa. O objetivo deste estudo foi relatar a experiência do tratamento tópico das lesões decorrentes da Síndrome de Stevens Johnson, utilizando-se membrana de celulose porosa e ácidos graxos essenciais. Trata-se de um relato de caso sobre o cuidado instituído a um paciente com lesões, cujos dados evolutivos foram obtidos por meio da avaliação dos registros do prontuário e da avaliação fotográfica. Paciente com 33 anos, sexo feminino, apresentava flictenas e placas eritematosas em toda extensão corpórea. Utilizaram-se, para cobertura das lesões, a membrana de celulose porosa e os ácidos graxos essenciais. O tratamento tópico utilizado promoveu alívio da dor, visualização adequada da lesão, drenagem espontânea e controle da infecção, manutenção da umidade local, diminuição ou ausência das trocas de curativo e hidratação da pele. No $15^{\circ}$ dia de tratamento, obteve-se epitelização parcial das lesões, e a paciente foi encaminhada para acompanhamento ambulatorial. A terapia tópica utilizada foi adequada neste caso, resultando em evolução satisfatória das lesões.
\end{abstract}

DESCRITORES: Estomaterapia. Enfermagem. Cicatrização de feridas. Síndrome de Stevens-Johnson.

\begin{abstract}
Stevens-Johnson Syndrome can be defined as an acute inflammatory, febrile, and self-limited disease that affects the skin and mucous membrane. The aim of this study was to report the experience of topical treatment of injuries from Stevens-Johnson Syndrome, using porous cellulose membrane and essential fatty acids. This is a case report about the care provided to a patient with injuries from Stevens-Johnson Syndrome. The outcome data were obtained by evaluating records and photographic assessment of the patient. Patient aged 33 years, female, with blisters and erythematous plaques throughout body extension. The porous cellulose membrane and the essential fatty acids were used for wound coverage. The topical treatment promoted pain relief, adequate lesion visualization, spontaneous drainage and infection control, maintenance of local humidity, decrease or absence of dressing changes and skin hydration. On the $15^{\text {th }}$ day of treatment, there was partial epithelialization of lesions and the patient was referred to an outpatient clinic. The topical therapy used was appropriate in this case, resulting in a satisfactory evolution of the injuries.
\end{abstract}

DESCRIPTORS: Stomatherapy. Nursing. Wound healing. Stevens-Johnson Syndrome.

'Universidade Federal de Santa Maria (UFSM) - Santa Maria (RS), Brasil. Endereço para correspondência: Rua Cipreste, 385 - Parque Verde -

CEP. 85807-700 - Cascavel (PR),Brasil -E-mail: anacasarolli@hotmail.com

¿Universidade do Oeste do Paraná (UNIOESTE) - Cascavel (PR), Brasil.

¿Universidade Federal do Paraná (UFPR) - Curitiba (PR), Brasil.

${ }^{4}$ UNIOESTE - Cascavel (PR), Brasil.

5UFSM - Santa Maria (RS), Brasil.

Artigo recebido em: 13/11/2014 - Aceito para publicação em: 28/06/2015 


\section{RESUMEN}

El síndrome de Stevens Johnson puede ser definido como una enfermedad inflamatoria aguda, febril, auto-limitante, que afecta a la piel y a la membrana mucosa. El objetivo de este estudio fue describir la experiencia del tratamiento tópico de las lesiones provocadas por el Síndrome de Stevens Johnson, con el uso de membrana de celulosa porosa y ácidos esenciales. Se trata de un caso acerca del cuidado establecido al paciente con lesiones, cuyos datos de evolución se obtuvieron mediante el análisis de los registros de las historias clínicas y la evaluación fotográfica. Paciente con 33 años, de sexo femenino, tenía burbujas y placas eritematosas en toda la extensión del cuerpo. Para cubrir las lesiones, fueron utilizadas la membrana de celulosa porosa y los ácidos grasos esenciales. El tratamiento tópico utilizado promovió el alivio del dolor, la visualización adecuada de la lesión, el drenaje espontáneo y el control de la infección, el mantenimiento de la humedad local, la disminución o ausencia de cambios de apósito y la hidratación de la piel. En el día 15 de tratamiento, se obtuvo epitelización parcial de las lesiones, y la paciente fue referida a un ambulatorio. La terapia tópica utilizada fue apropiada en este caso, lo que resultó en la evolución satisfactoria de las lesiones.

DESCRIPTORES: Estomaterapia. Enfermería. Cicatrización de heridas. Síndrome de Stevens-Johnson.

\section{INTRODUÇÃO}

A Síndrome de Stevens Johnson (SSJ) consiste em uma patologia mucocutânea que corresponde a uma reação de hipersensibilidade tardia, pouco frequente, porém, potencialmente fatal, afetando principalmente adultos jovens. A maioria dos casos está relacionada com o uso de fármacos, embora determinadas infecções também possam desencadear o problema ${ }^{1,2}$.

Em face da gravidade da SSJ e a lacuna na literatura de Enfermagem sobre este assunto, tornou-se relevante a realização deste relato. Diante do exposto, o objetivo foi relatar a experiência do tratamento tópico de lesões decorrentes da síndrome de Stevens Johnson, com o uso da membrana de celulose porosa e dos ácidos graxos essenciais.

\section{MÉTODO}

Trata-se de um relato de caso de uma paciente com SSJ, internada na unidade de neurologia e ortopedia de um hospital universitário do Paraná. Os dados foram coletados por meio de avaliação direta das lesões, dos registros escritos no prontuário da paciente e por registros fotográficos, com consentimento documentado da mesma. O projeto de pesquisa foi aprovado pelo Comitê de Ética em Pesquisa da Universidade Estadual do Oeste do Paraná, sob parecer 487.554.

\section{RESULTADOS}

Paciente com 33 anos, sexo feminino, admitida na instituição em junho de 2013, referindo quadro de mal-estar geral, coriza, congestão nasal e febre, fazendo uso de dipirona sódica. Relatava ter transtorno bipolar e fazer uso de antipsicóticos, tranquilizantes, neurolépticos e antiepiléticos. A participante foi diagnosticada com SSJ em 15 de junho de 2013, em função do uso concomitante de lamotrigina e dipirona sódica, sendo tratada com hidrocortisona.

Em 17 de junho de 2013, solicitou-se avaliação da Comissão de Curativos da instituição. Ao exame físico, apresentava flictenas e placas eritematosas em face (Figura 1), pavilhão auricular, tórax anterior e posterior, região genital e membros superiores e inferiores. Cavidade oral apresentando lesões aftoides e vesiculares. Iniciou-se terapia tópica diária, realizando-se limpeza das lesões com solução fisiológica $0,9 \%$ aquecida, aspiração do exsudato de flictenas íntegras, desbridamento mecânico de tecido frouxo com jatos de solução fisiológica e com instrumental cortante. Em seguida, aplicou-se curativo da membrana de celulose porosa nas regiões com perda de tecido superficial e ácidos graxos essenciais em toda a extensão corpórea, sem uso de cobertura secundária. As roupas de cama utilizadas haviam passado por processo de esterilização. $\mathrm{O}$ curativo foi renovado diariamente.

Após quatro dias, notou-se melhora importante no aspecto das lesões e melhora progressiva da dor, sem aparecimento de novas flictenas (Figura 2). A pele perilesional apresentava sinal de Nikolsky positivo, traduzido pelo descolamento da epiderme quando se exerce uma pressão digital tangencial. Após 15 dias, a lesão apresentava epitelização parcial sem presença de novas flictenas, com a realização apenas de hidratação com ácidos graxos essenciais. Essa terapia acarretou melhora do aspecto das lesões e da dor (Figura 3). 


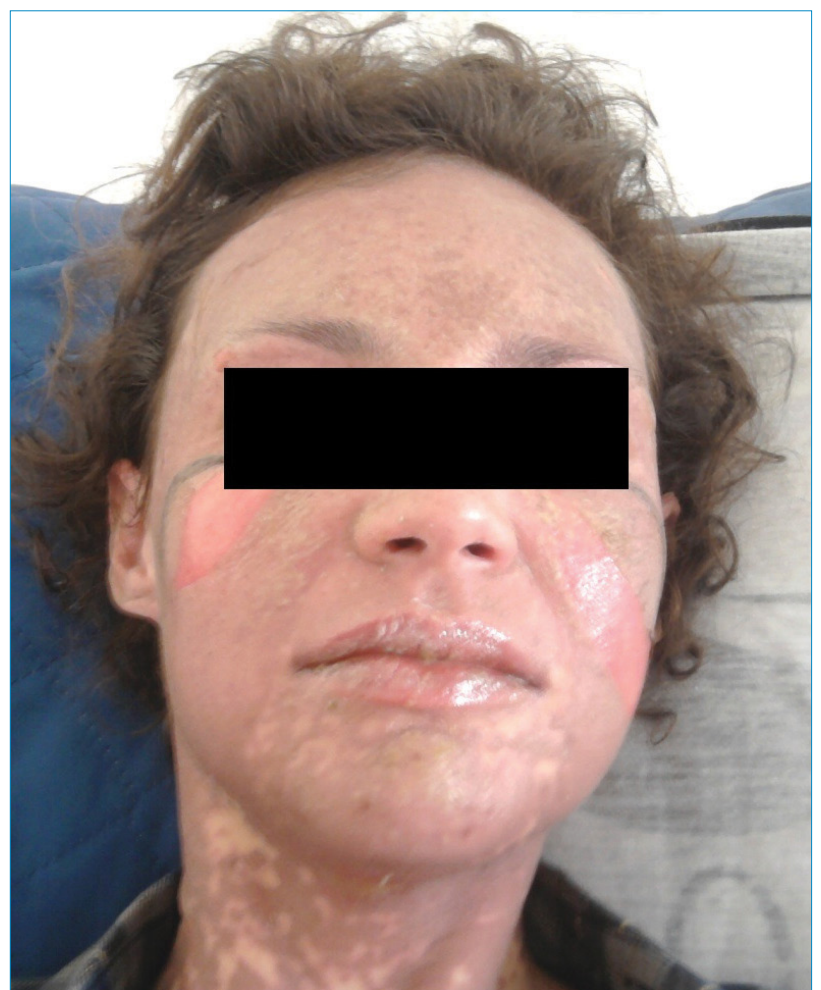

Figura 1. Primeira avaliação.

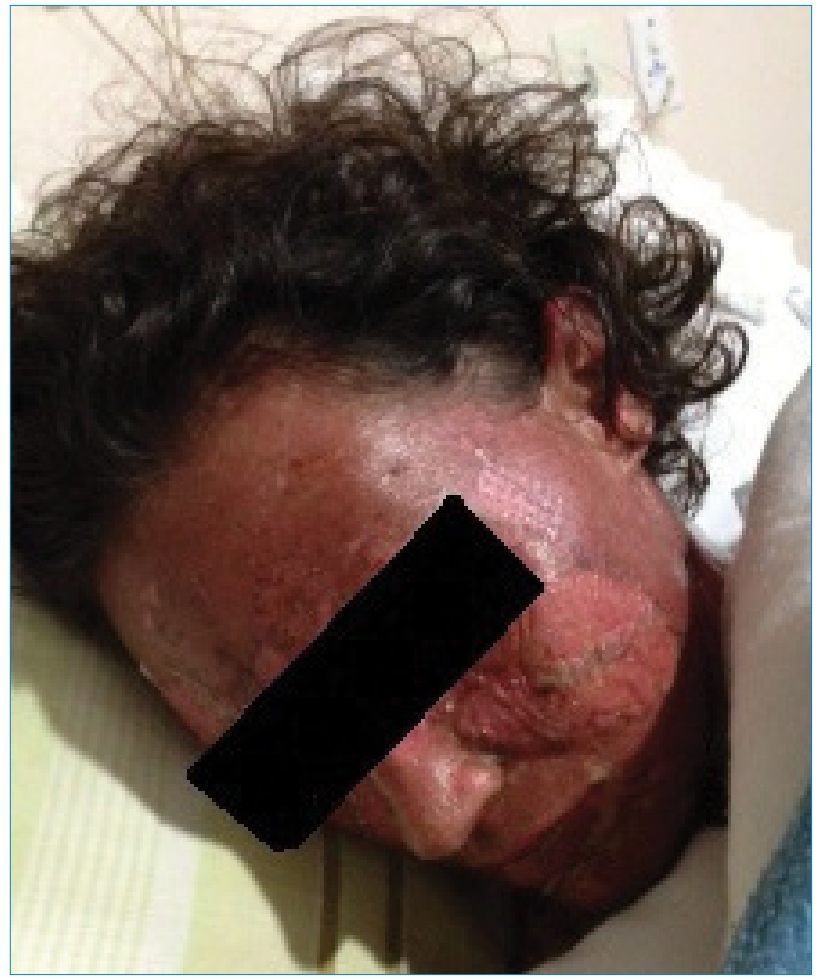

Figura 2. Quarto dia após início da terapia.

\section{DISCUSSÃO}

A avaliação de Enfermagem ao paciente com lesões deve ser realizada de forma sistemática, individualizada e integral, de modo a identificar e tratar não só as manifestações cutâneas, como também as necessidades psicológicas, sociais, biológicas e culturais.

Não existe um consenso, na literatura, sobre o tratamento tópico destes pacientes. No entanto, a manipulação deve ser cuidadosa e asséptica, além de criar campo estéril $1^{3}$. Porém, o tratamento tópico utilizado baseou-se nos princípios fundamentais do tratamento de feridas e na utilização de coberturas adequadas ao processo de cicatrização e controle de infecção.

A membrana de celulose é um biomaterial que possui propriedades vantajosas no tratamento de lesões, tais como facilidade na aplicação, excelente adesão aos tecidos, diminuição da dor, visualização adequada do leito da ferida, drenagem espontânea, resistência, afinidade à água, diminuição ou ausência das trocas de

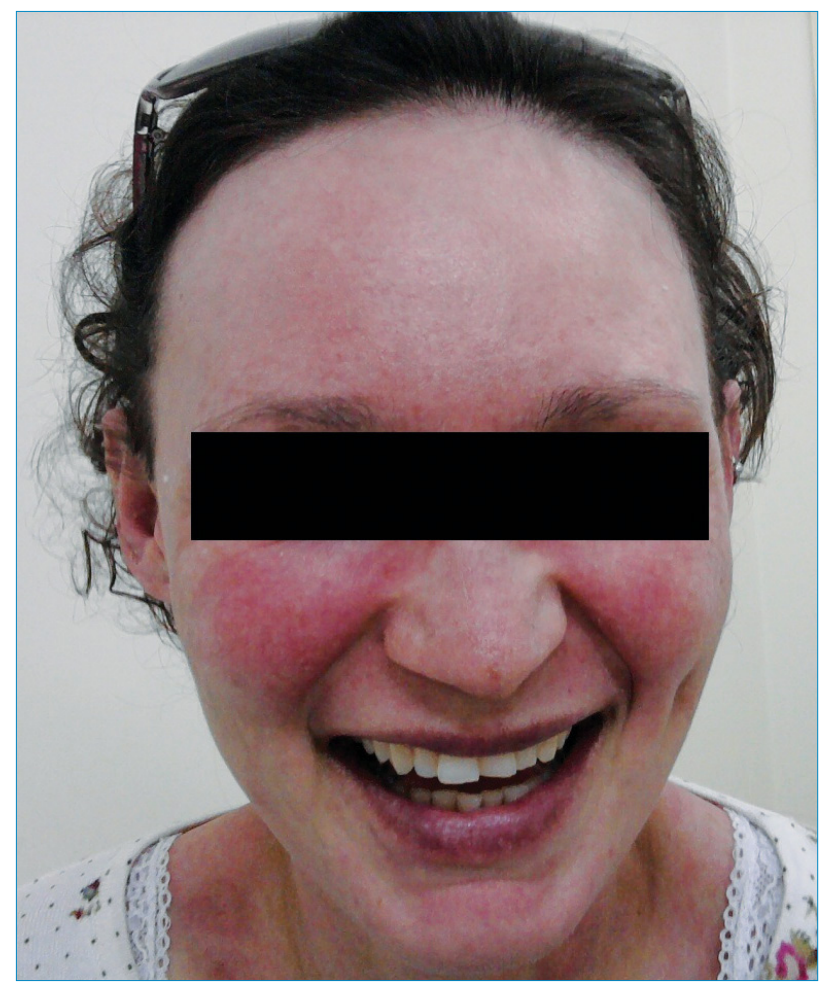

Figura $3.15^{\circ}$ dia após início da terapia. 
curativos e aumento no intervalo do acompanhamento do profissional $1^{4,5}$.

Os ácidos graxos essenciais formam uma barreira protetora da pele, impedindo a maceração, além de serem importantes no processo de inflamação celular, proporcionando alívio após a primeira aplicação e nutrição celular local, além de grande capacidade de regeneração dos tecidos ${ }^{6}$. Além disso, o uso de ácidos graxos essenciais mostrou-se como o tratamento tópico mais utilizado nos pacientes com SSJ, em estudo transversal realizado em hospital no Distrito Federal7.

\section{CONCLUSÕES}

O presente estudo ampliou o conhecimento e a experiência sobre o tratamento de lesões cutâneas provocadas por SSJ. A literatura sobre o assunto apresenta-se escassa, requerendo a aplicação de conhecimentos adquiridos com outros tipos de lesões, considerando a avaliação individual da paciente. Foram respeitados os princípios fundamentais do tratamento de feridas, que levaram ao favorecimento da cicatrização e à redução da dor, utilizando a terapia tópica relatada.

\section{REFERÊNCIAS}

1. Oliveira A, Sanches HE, Selores M. O espectro clínico Síndrome de Stevens Johnson e necrólise epidérmica tóxica. Acta Med Port. 2001;24(4):995-1002.

2. Ensina LF, Fernandes FR, Di Gesu G, Malaman MF, Chavarria ML, Bernd LA. Reações de Hipersensibilidade a Medicamentos - Parte III. Rev Bras Alergia Imunopatol. 2009;32(5):178-83.

3. Bulisani AC, Sanches GD, Guimarães HP, Lopes RD, Vendrame LS, Lopes AC. Síndrome de Stevens-Johnson e necrólise epidérmica tóxica em medicina intensiva. Rev Bras Ter Intensiva. 2006;18(3):292-7.

4. Yaguishita N. Cicatrização induzida pela membrana de celulose porosa (Membracel ${ }^{\circledR}$ ) em dorso de ratos. [dissertação]. Curitiba, PR: Faculdade Evangélica do Paraná; 2006.
5. Vieira JC, Badin AZ, Calomeno LH, Teixeira V, Ottoboni E, Bailak $M$, et al. Membrana porosa de celulose no tratamento de queimaduras. Arq Catarinenses Med. 2007;36(Suppl 1):94-7.

6. Ferreira AM, Souza BM, Rigotti MA, Loureiro MR. Utilização dos ácidos graxos no tratamento de feridas: uma revisão integrativa da literatura nacional. Rev Esc Enferm. 2012;46(3):752-60.

7. Emerick MF, Rodrigues MM, Pedrosa DM, Novaes MR, Gottems L. Síndrome de Stevens-Johnson e Necrólise Epidérmica Tóxica em um hospital do Distrito Federal. Rev Bras Enferm. 2014;67(6):898-904. 